\title{
Photogrammetry-based VR Interactive Pedagogical Agent for K12 Education
}

\author{
Laduona Dai \\ L.Dai_1@tilburguniversity.edu \\ Tilburg University \\ Netherlands
}

\begin{abstract}
The effects of using various multimedia pedagogical agents with computer screens have been extensively studied in the past. However, two topics in this area of research remain relatively unexplored: the impact of virtual reality and of real-identity agents with high human likeness. The goal of my $\mathrm{PhD}$ project is to build and evaluate virtual reality agents that resemble well-known experts or role models in the learning content domain for K12 students. Three sub-systems necessary to build the pedagogical agent will be constructed and evaluated: speech synthesis, facial movement generation, and dialogue system. The different stages and the accompanying methodologies are outlined in this paper, including an overview of the deliverables.
\end{abstract}

\section{CCS CONCEPTS}

- Human-centered computing $\rightarrow$ Virtual reality; • Social and professional topics $\rightarrow \mathrm{K}-12$ education.

\section{KEYWORDS}

pedagogical agents, virtual reality, multimedia learning, learning effectiveness, text-to-speech

\section{ACM Reference Format:}

Laduona Dai. 2021. Photogrammetry-based VR Interactive Pedagogical Agent for K12 Education. In Proceedings of the 2021 International Conference on Multimodal Interaction (ICMI '21), October 18-22, 2021, Montréal, QC, Canada. ACM, New York, NY, USA, 4 pages. https://doi.org/10.1145/3462244. 3481280

\section{INTRODUCTION}

Multimedia pedagogical agents have been used as tools for education since the late 1990s [20,24]. Many studies have evaluated how agent characteristics affect students' learning outcomes [3, 6, 22]. The most commonly evaluated agent features are (2D or 3D) format, appearance, voice, facial expressions and body gestures. Overall, the majority of the studies have focused on examining the effects of fictional agents presented on traditional computer screens in $2 \mathrm{D}$ or 3D formats. However, in the last decade, we have witnessed a rapid development of virtual reality (VR) solutions. VR - particularly the VR that includes the usage of head-mounted-displays (HMD) can provide users with the feeling of "being there" and thereby provide

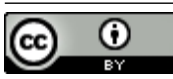

This work is licensed under a Creative Commons Attribution International 4.0 License.

ICMI '21, October 18-22, 2021, Montréal, QC, Canada

(c) 2021 Copyright held by the owner/author(s).

ACM ISBN 978-1-4503-8481-0/21/10.

https://doi.org/10.1145/3462244.3481280 potential benefits for learning. In particular, compared to conventional display, the feeling of presence provided by immersive VR could potentially remove physical limitations and let learners submerge in the virtual environment. Despite the fact that past studies have shown various benefits of using VR for learning, VR does not appear to be widely used for pedagogical agent research. Yet it could help the learner with more engagement and improve their cognitive skills [12] and it might be a good modality for learning abstract or conceptual knowledge, as well as procedural skill-related content [10].

Next to the VR solutions, another area of pedagogical agent research that remains relatively unexplored is the use of realisticidentity agents with high human likeness. To our knowledge, almost all studies on the usage of pedagogical agents choose for fictional agent roles instead of using real identify agents, for example, famous scientists or historical figures. The use of real-world characters could have a positive impact on learning due to the agent being perceived as an expert, as well as due to an increased engagement of the user triggered by the accompanying narrative. At this point, though, it is unclear what the learning effects are when using a VR agent with an authentic voice and appearance modelled after a real person who possesses expert authority or is viewed as a role model in the content field. Since recorded videos of real humans lack interactive features, speech synthesis and photogrammetry technology could be utilized to replicate a real person in VR. Therefore, the general aim of this project is to explore how a VR agent with high human likeness modelled on a real-world expert or a role model could help students' improve their learning gains.

\section{MOTIVATION AND PROBLEM STATEMENT}

In this $\mathrm{PhD}$ project, we will build and evaluate the effects of photogrammetry based VR pedagogical agents on K12 students' learning outcomes and motivation using existing learning materials and test questions. The objective is to improve learning gains by using realistic-looking and interactive VR agents that resemble wellknown experts or role models in the learning content domains.

Thanks to the advances of text-to-speech (TTS), computer vision and natural language processing, it is now possible to synthesize a specific individual voice, create dynamic 3D facial movements and build interactive dialogue systems. Some of these technologies have been used in the past studies on pedagogical agents, for example, using generic voice from commercial TTS engine and synchronizing facial movements from speech. To the best of our knowledge there is however no study that has evaluated the effects of pedagogical agents that includes all these features, i.e., 1) an interactive dialogue VR agent that has the appearance of a role model 2) with generated facial movements from 3D photo scans and 3) synthesized speech 
replicating the original voice. Thus, to understand how such an agent affects K12 student learning outcomes, motivation and perception of the agent, we face the following challenges: training TTS models that could produce speech for desired voices, creating 3D facial movements with synchronization to speech using static neutral face photogrammetry scans, and integrating an agent dialogue system that can be adapted to certain learning content.

\section{EXAMPLE USE CASE}

The target use case for this research project is a VR pedagogical agent that would be used inside and outside of a classroom for K12 students. The idea of this implementation is to promote learning with the guidance of an expert (the VR agent) who is a known personality in the student's country. For example, an agent appears in an astronaut outfit with the face of a real astronaut's face. The objective is to build photo-realistic and interactive VR agents that look and sound like real experts or role models by using speech synthesis and photogrammetry technology.

\section{RESEARCH QUESTIONS}

Derived from motivation and problem statement in the previous section, this $\mathrm{PhD}$ project aims to answer the following research questions:

RQ1: How do K12 students perceive photogrammetry-based VR interactive pedagogical agent with synthesized speech and dynamic facial animations?

To answer this question, we aim to evaluate several different variables during the process of building a complete agent: the naturalness of the TTS and facial animations, the effects of agent identity, and the usability of the agent dialogue system.

RQ2: How does the usage of this type of VR pedagogical agents affect K12 students' learning outcomes and motivation?

Depending on the types of knowledge, treatment duration and frequency, the learning effects of using pedagogical agents could vary Since we will use agents that are VR version well-known human experts or role models in the content domain, we expect the usage of this kind of agents will positively affect students' motivation. In order to test this assumption, we aim to compare the use of a VR agent to conventional classroom teaching.

\section{RELATED WORK}

In this section, we present a brief summary of related works about text-to-speech (TTS) systems, facial movement generation and dialogue systems.

\subsection{TTS systems}

Modern TTS systems are sequence-to-sequence architectures, which means that the inputs are text sentences and outputs are wave forms of speech. These systems have two main components: a feature prediction network and a vocoder network. The feature prediction network converts text embeddings into Mel-scale spectrograms, and the vocoder network produces time-domain waveforms using
Mel-scale spectrograms. A typical example of a modern TTS system is Tacotron 2 by Google [23]. The model appears to synthesize speech with a mean opinion score (MOS) of 4.53 out of 5 , which is relatively high compared to the score for recorded human speech of 4.58. More recent studies on TTS systems have focused on synthesizing more natural and robust speech [15], training the model with limited amount of materials $[5,13]$, and synthesizing multilingual speech [18, 27]. However, studies [4] have pointed out that the style of the training material could affect the perceived naturalness of synthesized speech; for example, news speech materials are better than conversational and book speech. They also found that speech quality of TTS models that target replicating certain voices are overall better than the quality of voice conversion models that aim at producing different voice from the original speaker.

\subsection{Facial movement generation}

To generate facial movement for a realistic VR agent with a high likeness to an existing person, it is necessary to use rigging techniques to reconstruct the virtual face and generate facial expressions. There are generally two different approaches for face reconstruction, either using RGBD sensors (e.g., [2]) or using multi-camera arrays (e.g., [1]). In this study, we will use the photogrammetry method with multi-camera arrays to acquire a high-quality static face model. For dynamic facial animations, the common method is to use linear blendshape models [14]. To generate facial animations with this method, a set of FACS [7] blendshapes is required. However, acquiring the FACS blendshapes is not an easy task, since they are either constructed by scanning real human while displaying corresponding expressions or crafted manually. A more convenient method that emerged in recent years is to generate the expression blendshapes for the target face using existing sample facial rigs (e.g., [26]). The advantage of this method is that it only requires a neutral face scan as input.

\subsection{Dialogue systems}

Dialogue systems can be categorized as either task-oriented or opendomain, depending on their application purpose. Task-oriented systems deal with specific topics in a certain content domain (e.g., a ticket booking chatbot), while open-domain systems are usually designed to maximize user engagement [11]. For the purposes of our project, we will focus on task-oriented systems. Based on the architectures, we can further categorize task-oriented systems into the pipeline and end-to-end methods. The pipeline systems consist of four different modules: natural language understanding (NLU), dialogue state tracking (DST), dialogue policy and natural language generation (NLG). NLU converts the user's input into structured semantic representations, DST tracks the dialogue states with the user's current input and dialogue history, dialogue policy decides the system's next action based on the current dialogue state, and NLG produces the output in natural language according to the action policy [19]. The end-to-end system utilizes one neural model to perform input understanding and output generation at the same time. Both pipeline and end-to-end methods have their pros and cons. In pipeline methods, different modules are trained separately, the good performance of each module does not guarantee the whole system works well [8], and the training materials require annotated 
datasets, which are human labour-intensive to construct. However, due to the separate module function design, pipeline systems are more transparent and controllable than just one neural model. The end-to-end methods could optimize the whole system as one unit, but require a large amount of training materials and difficult to check the underlining decisions.

\section{RESEARCH PLAN AND METHODOLOGY}

This $\mathrm{PhD}$ project is planned for 4 years (September, 2020 to September, 2024) with four stages. After a systematic literature review is conducted, 3 sub-systems for the agent will be built and tested one by one.

\subsection{Stage 1: Systematic literature review}

Before directly building the pedagogical agent, the first stage of this research would be conducting a systematic literature review about studies in the last decade that use agents for learning. The aims of the review are to: (1) Provide an overview of the design features for the agents and their impact on learning; (2) Collect technical information on how different agents are created with focus on audio and visual features; (3) Describe the experimental and contextual conditions used in past studies with pedagogical agents; and (4) Understand how past studies conducted evaluations of learning outcomes, learner motivation and agent perception. The results of the literature review will be used as a reference when we build and evaluate our agent.

\subsection{Stage 2: Dutch TTS model}

In the second stage of the research, we plan to train a Dutch TTS model for our desired use case. As a starting point, we will use the Tacotron 2 architecture since it has been implemented by many Python libraries. In the Tacotron 2 paper by Google [23], the quality of their synthesized speech was achieved by using an internal English dataset with around 25 hours of materials. From a practical perspective, it may be difficult to acquire 25 hours of high quality recorded speech by any given real-life personality. Thus, we would first use other Dutch speech materials to investigate what type of speech materials are best for training a TTS model and what the minimum amount of training material is needed for training a Dutch TTS model with acceptable quality. To evaluate the quality of synthesized speech, a user study will be conducted with our target population by examining the quality and perception of the custom voice. All user studies in the project will be conducted in line with our institutional ethical review board requirements and procedures.

\subsection{Stage 3: Facial movement generation for the VR agent}

Instead of developing a new model for facial movement generation with synchronization to speech, we plan to use available architectures that could be adapted to our project. So the first step in this stage would be investigating and comparing all up-to-date models that support 3D facial movement generation and emotional expressions in real-time using our training data. After evaluating the models for their quality and flexibility, we will then train the most suitable model using the 3D scans of a neutral face. For the evaluation of this sub-system, we could first compare agents that using generated facial movements with real-human recorded videos, in both scenarios the voice would be the same real-human recorded speech. Then the Dutch TTS model developed in stage 2 will be integrated and we will examine how the target population perceives the agent, comparing recorded and synthesized modalities. If time allows, we will also consider adding body gestures since they can complement the information conveyed by facial expressions.

\subsection{Stage 4: Agent dialogue system}

Similar to the previous stage, we will also use readily available technology that could be modified and improved for the agent dialogue system. The dialogue system will be trained using materials for specific learning content and provide possibilities to be controlled by humans in specific exchanges. This is because most neural-network based dialogue systems are a "black box" - the process for generating conversations is not transparent and might lead to inappropriate responses to user requests. Thus, we will first evaluate which dialogue systems meet our requirements and then examine those features with a pre-test before integrating this subsystem into the agent. Finally, when the dialogue system is chosen and evaluated, we will combine all three sub-systems and conduct experiments on how the full VR pedagogical agent affects student learning outcomes and motivation.

\section{COMPLETED AND PLANNED RESEARCH}

As planned for the first stage of the research, a systematic literature review about using pedagogical agents in education has been conducted. The coding for each study about agent design characteristics, experiment contextual features and results evaluation has been completed. Preliminary results of the review provided a number of insights and helped us identify research gaps in the literature. Importantly, a clear majority of the existing studies provides outcomes for non-VR agents, with only a few studies examining the use of VR pedagogical agents (e.g., $[9,16,21,25])$. Next to that, among all the studies included for the review, none of them used photogrammetry to build the agent. Most agents have the format of a 3D humanoid with a cartoon-like appearance. For studies that reported the usage and format of the speech, most of them chose to use recorded human speech instead of synthesized speech. All the studies with synthesized speech utilized available voices from their chosen TTS engine; no study created a custom voice for the agent. Interestingly, only one study evaluated the effects of using an agent that was modelled after a well-known person in the learning content domain [9]. The agent had the face resembling Neil Armstrong in an astronaut outfit while demonstrating the Apollo 11 mission. However, the voice of the agent was a generic one available from the TTS engine, rather than Neil Armstrong's voice. Finally, there is an underrepresentation of studies that focus on the K12 population. Only $9 \%$ of our included studies that reported population age researched K12 students. The majority examined how pedagogical agents affect student learning at middle school or university level.

Apart from the systematic review, early experiments on training a Dutch TTS model have also been conducted. By training a Tacotron 2 model using 20 hours of audiobook materials, we have 
verified that the chosen TTS architecture could indeed produce good quality Dutch speech. We used the following library for the Tacotron 2 architecture: Mozilla TTS [17]. We gained some firsthand experience of the conditions for good training materials. For example, 7 hours of training materials were not enough to train a decent quality model when using Tacotron 2 . We also concluded that the speaker's voice for the training materials should have a neutral tone, minimal intonation and impersonation, and no mix of multiple languages.

Currently, we are finalizing the writing of the systematic literature review paper. For the next steps, we will first conduct more experiments to find out what the minimum required amount of material is that is required to train a decent quality Dutch TTS model. We plan to evaluate the quality of synthesized speech in September or October this year with our target population.

\section{EXPECTED CONTRIBUTION}

This proposed research project aims to contribute to the pedagogical agent field in the following ways:

\section{Artifact contribution}

- Building photogrammetry-based VR interactive agent with real identity using speech synthesis and dynamic facial animations.

\section{Empirical contribution}

- Evaluating the effects of using VR pedagogical agents for the under-representative population (K12 students) in the research field.

- Providing insights about how students perceive a photorealistic VR agent with the identity of a well-known human expert or role model in the learning content domain.

- Examining the effects on learning and motivation when using a real-identity VR pedagogical agent.

\section{ACKNOWLEDGMENTS}

I would like to thank my advisors Prof. Max Louwerse, Dr. Marie Postma and Dr. Merel Jung for their guidance and supervision in this $\mathrm{PhD}$ project. This research is part of the MasterMinds project, funded by the RegionDeal Mid- and West-Brabant, and is co-funded by the Ministry of Economic Affairs and Municipality of Tilburg.

\section{REFERENCES}

[1] Jascha Achenbach, Eduard Zell, and Mario Botsch. 2015. Accurate Face Reconstruction through Anisotropic Fitting and Eye Correction. In Vision, Modeling \& Visualization, David Bommes, Tobias Ritschel, and Thomas Schultz (Eds.). The Eurographics Association. https://doi.org/10.2312/vmv.20151251

[2] Chen Cao, Yanlin Weng, Shun Zhou, Yiying Tong, and Kun Zhou. 2014. FaceWarehouse: A 3D Facial Expression Database for Visual Computing. IEEE transactions on visualization and computer graphics 20 (03 2014), 413-25. https: //doi.org/10.1109/TVCG.2013.249

[3] Juan Castro-Alonso, Rachel Wong, Olusola Adesope, and Fred Paas. 2021. Effectiveness of Multimedia Pedagogical Agents Predicted by Diverse Theories: a Meta-Analysis. Educational Psychology Review (01 2021), 1-27. https: //doi.org/10.1007/s10648-020-09587-1

[4] Erica Cooper and Junichi Yamagishi. 2021. How do Voices from Past Speech Synthesis Challenges Compare Today? arXiv:2105.02373 [cs.SD]

[5] Ander Corral, Igor Leturia, Aure Séguier, Michäel Barret, Benaset Dazéas, Philippe Boula de Mareüil, and Nicolas Quint. 2020. Neural Text-to-Speech Synthesis for an Under-Resourced Language in a Diglossic Environment: the Case of Gascon Occitan. In Proceedings of the 1st foint Workshop on Spoken Language Technologies for Under-resourced languages (SLTU) and Collaboration and Computing for
Under-Resourced Languages (CCURL). European Language Resources association, Marseille, France, 53-60. https://www.aclweb.org/anthology/2020.sltu-1.8

[6] Robert O. Davis. 2018. The impact of pedagogical agent gesturing in multimedia learning environments: A meta-analysis. Educational Research Review 24 (2018), 193-209. https://doi.org/10.1016/j.edurev.2018.05.002

[7] Paul Ekman and Wallace Friesen. 1978. Facial action coding system: a technique for the measurement of facial movement.

[8] Jianfeng Gao, Michel Galley, and Lihong Li. 2018. Neural Approaches to Conversational AI. In Proceedings of the 56th Annual Meeting of the Association for Computational Linguistics: Tutorial Abstracts. Association for Computational Linguistics, Melbourne, Australia, 2-7. https://doi.org/10.18653/v1/P18-5002

[9] Foteini Grivokostopoulou, Konstantinos Kovas, and Isidoros Perikos. 2020. The effectiveness of embodied pedagogical agents and their impact on students learning in virtual worlds. Applied Sciences (Switzerland) 10, 5 (2020). https://doi.org/10.3390/app10051739

[10] David Hamilton, Jim McKechnie, Edward Edgerton, and Claire Wilson. 2021. Immersive virtual reality as a pedagogical tool in education: a systematic literature review of quantitative learning outcomes and experimental design. Fournal of Computers in Education 8, 1 (Mar 2021), 1-32. https://doi.org/10.1007/s40692020-00169-2

[11] Minlie Huang, Xiaoyan Zhu, and Jianfeng Gao. 2020. Challenges in Building Intelligent Open-domain Dialog Systems. ACM Transactions on Information Systems 38 (04 2020), 1-32. https://doi.org/10.1145/3383123

[12] Lasse Jensen and Flemming Konradsen. 2018. A review of the use of virtual reality head-mounted displays in education and training. Education and Information Technologies 23 (07 2018), 1-15. https://doi.org/10.1007/s10639-017-9676-0

[13] Roee Levy-Leshem and Raja Giryes. 2021. Taco-VC: A Single Speaker Tacotron based Voice Conversion with Limited Data. In 2020 28th European Signal Processing Conference (EUSIPCO). 391-395. https://doi.org/10.23919/Eusipco47968.2020. 9287448

[14] J. P. Lewis, Ken Anjyo, Taehyun Rhee, Mengjie Zhang, Frédéric H. Pighin, and Zhigang Deng. 2014. Practice and Theory of Blendshape Facial Models. In Eurographics.

[15] Naihan Li, Yanqing Liu, Yu Wu, Shujie Liu, Sheng Zhao, and Ming Liu. 2020. RobuTrans: A Robust Transformer-Based Text-to-Speech Model. Proceedings of the AAAI Conference on Artificial Intelligence 34, 05 (Apr. 2020), 8228-8235. https://doi.org/10.1609/aaai.v34i05.6337

[16] Guido Makransky, Philip Wismer, and Richard E. Mayer. 2019. A gender matching effect in learning with pedagogical agents in an immersive virtual reality science simulation. Fournal of Computer Assisted Learning 35, 3 (2019), 349-358. https: //doi.org/10.1111/jcal.12335

[17] Mozilla. 2021. TTS. https:/github.com/mozilla/TTS

[18] Tomáš Nekvinda and Ondřej Dušek. 2020. One Model, Many Languages: MetaLearning for Multilingual Text-to-Speech. In Proc. Interspeech 2020. 2972-2976. https://doi.org/10.21437/Interspeech.2020-2679

[19] Jinjie Ni, Tom Young, Vlad Pandelea, Fuzhao Xue, V. Adiga, and E. Cambria. 2021. Recent Advances in Deep Learning Based Dialogue Systems: A Systematic Survey. ArXiv abs/2105.04387 (2021).

[20] Ana Paiva and Isabel Machado. 1998. Vincent, an Autonomous Pedagogical Agent for On-the-Job Training (ITS '98). Springer-Verlag, Berlin, Heidelberg, 584-593.

[21] Susanne Schmidt, Gerd Bruder, and Frank Steinicke. 2019. Effects of virtual agent and object representation on experiencing exhibited artifacts. COMPUTERS and GRAPHICS 83 (Dec 2019), 1-10. https://doi.org/10.1016/j.cag.2019.06.002

[22] Noah Schroeder, Olusola Adesope, and Rachel Gilbert. 2013. How Effective are Pedagogical Agents for Learning? A Meta-Analytic Review. fournal of Educational Computing Research 49 (07 2013), 1-39. https://doi.org/10.2190/EC.49.1.a

[23] Jonathan Shen, Ruoming Pang, Ron J. Weiss, Mike Schuster, Navdeep Jaitly, Zongheng Yang, Zhifeng Chen, Yu Zhang, Yuxuan Wang, Rj Skerrv-Ryan, Rif A. Saurous, Yannis Agiomvrgiannakis, and Yonghui Wu. 2018. Natural TTS Synthesis by Conditioning Wavenet on MEL Spectrogram Predictions. In 2018 IEEE International Conference on Acoustics, Speech and Signal Processing (ICASSP). 4779-4783. https://doi.org/10.1109/ICASSP.2018.8461368

[24] Brian A. Stone and James C. Lester. 1997. Dynamically Sequencing an Animated Pedagogical Agent. Morgan Kaufmann Publishers Inc., San Francisco, CA, USA, 156-163.

[25] Tzu-Yu Tai, Howard Hao-Jan Chen, and Graeme Todd. 2020. The impact of a virtual reality app on adolescent EFL learners' vocabulary learning. COMPUTER ASSISTED LANGUAGE LEARNING (2020). https://doi.org/10.1080/09588221.2020. 1752735

[26] Mengjiao Wang, D. Bradley, Stefanos Zafeiriou, and Thabo Beeler. 2020. Facial Expression Synthesis using a Global-Local Multilinear Framework. Computer Graphics Forum 39 (05 2020), 235-245. https://doi.org/10.1111/cgf.13926

[27] Yu Zhang, Ron J. Weiss, Heiga Zen, Yonghui Wu, Zhifeng Chen, R.J. SkerryRyan, Ye Jia, Andrew Rosenberg, and Bhuvana Ramabhadran. 2019. Learning to Speak Fluently in a Foreign Language: Multilingual Speech Synthesis and Cross-Language Voice Cloning. In Proc. Interspeech 2019. 2080-2084. https: //doi.org/10.21437/Interspeech.2019-2668 